\title{
Detection and Tracking of the Pores of the Lamina Cribrosa in Three Dimensional SD-OCT Data
}

\author{
Florence Rossant ${ }^{1}$, Kate Grieve ${ }^{2}$, Stéphanie Zwillinger ${ }^{2}$, and Michel Paques ${ }^{2}$ \\ ${ }^{1}$ Institut Supérieur d'Electronique de Paris (ISEP), Paris, France \\ ${ }^{2}$ Clinical Investigation Center 1423, Quinze-Vingts Hospital, Paris, France \\ Florence.rossanteisep.fr, kategrieve@gmail.com, \\ stephanie.zwillinger@gmail.com, michel.paques@gmail.com
}

\begin{abstract}
Glaucoma is one of the leading causes of blindness in the world. Although its physiopathology remains unclear, the deformations of the lamina cribrosa (LC), a three-dimensional porous structure through which all the nerve fibers from the retina pass to join the brain, are very likely to play a major role. We present in this article a method for the $3 D$ reconstruction of the pores of the $L C$, i.e. of the axon pathways, from three dimensional SD-OCT data. This method is based on pore detection in one en-face plane and on pore tracking throughout the volume. To overcome difficulties due to the low signal to noise ratio, we model and integrate a priori knowledge regarding the structures to be segmented in all steps of our algorithm. The quantitative evaluation shows good results on a test set of 14 images, with 76\% of the axonal paths truly detected and an RMSE between the automatic and the manual segmentations around 2 pixels.
\end{abstract}

Keywords: Optical coherence tomography, 3D OCT, SD-OCT, retina, optic nerve, lamina cribrosa, tracking, pore segmentation.

\section{Introduction}

Glaucoma is one of the leading causes of blindness in the world. Although its physiopathology remains unclear, a major role of the deformations of the lamina cribrosa (LC), the structure located at the interface between the ocular globe and the optic nerve, is strongly suspected $[1,2]$. The LC is a three-dimensional porous structure composed of flexible collagenous tissue through which all the nerve fibers from the retina pass to join the brain. During glaucoma, it is likely that the deformations of the LC induce a deformation of the pores and hence damage bypassing neurons.

Optical Coherence Tomography (OCT) is a scanning ocular imaging technique that uses infrared light to observe the fine structures of the retina and the optic nerve. It offers the possibility of reconstructing in three dimensions the structures that are imaged. This led us to study the lamina cribrosa with this imaging modality, and in particular the 3D arrangement of the LC pores revealing the pathways of the axons.

Image processing methods have been already designed for the analysis of the LC from three dimensional OCT data. Algorithms for the automatic delineation of the LC from the surrounding structures [3] and for the 3D modeling of the LC anterior surface

adfa, p. 1, 2011.

(C) Springer-Verlag Berlin Heidelberg 2011 
[4,5] have been proposed. Moreover, Nadler [6] processed images acquired with two prototype OCT systems, in SS-OCT (Swept Source-OCT) and in multimodal imaging involving adaptive optics (MAO-OCT), in order to segment the pores in en-face scans extracted from the $3 \mathrm{D}$ data cube. However, no 3D reconstruction of the axonal pathways into the LC was achieved.

In this article, we present a method for the three-dimensional reconstruction and analysis of the pores of the LC from data acquired with SD-OCT (Spectral Domain OCT). To the best of our knowledge, this is the first attempt to achieve such a 3D model of the axonal pathways. The method has been applied in glaucomatous subjects to study the remodeling of the lamina cribrosa during glaucoma. We present in Section 2 the OCT data and in Section 3 the main features of the pores and their modeling. Section 4 introduces the main methods involved in the segmentation algorithm, which is detailed in Section 5. Experimental results are presented in Section 6.

\section{Three dimensional SD-OCT data}

3D OCT scans were acquired using a commercially available OCT apparatus (Spectralis ${ }^{\circledR}$ SD-OCT). An acquisition results in a set of 120 2D images each corresponding to a vertical or horizontal OCT slice (Fig. 1a). The resolution of each image (496 x 384) is about $12 \mu \mathrm{m}$ horizontally and $4 \mu \mathrm{m}$ vertically. The sampling step between two consecutive images is around $11 \mu \mathrm{m}$. The surface of the retina that is imaged is about $4.6 \times 1.3 \mathrm{~mm}^{2}$.

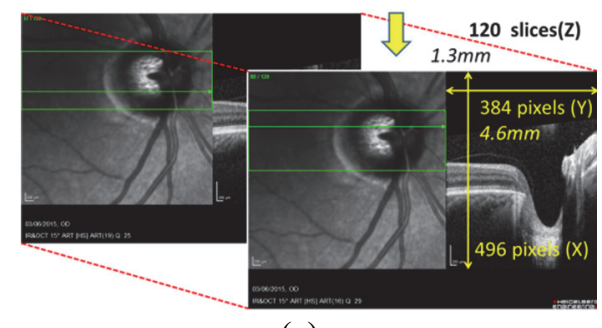

(a)

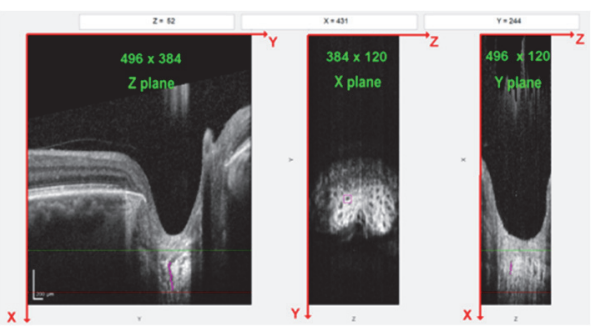

(b)

Fig. 1. 3D OCT data; (a) data made of 120 horizontal or vertical slices. The eye fundus image at the left-hand side shows the position of the slice, at the right hand side; (b) data displayed in 3 orthogonal planes: one en-face plane $\mathrm{X}$ and two transverse planes $\mathrm{Y}$ and $\mathrm{Z}$.

The 3D OCT data is extracted from this set of 120 images. We have developed a graphical interface that enables us to navigate inside the OCT cube and visualize it an an given point $(X, Y, Z)$. Three orthogonal $2 \mathrm{D}$ planes are displayed by fixing one of the coordinate (Fig. 1b). A horizontal plane of abscissa $X$ (called X plane in what follows) shows an en-face image, where the dark spots correspond to pores of the LC through which the axons pass. The higher the value of $X$, the deeper the slice inside the optic nerve. The axonal pathways appear as black curves in the two other planes, the $\mathrm{Y}$ and $\mathrm{Z}$ transverse planes. Our purpose is to initialize axon pathways (i.e. pores) in an X plane 
and to track them with respect to the increasing and decreasing X coordinates. This task is very difficult since the data is very noisy, and often low contrast. Moreover, the resolution is poor: only a few pixels form a pore in an X plane. Consequently, we propose a segmentation method that relies heavily on a priori knowledge, which is modeled and integrated in the pore detection and tracking procedures.

\section{$3 \quad$ Modeling a priori knowledge}

The gray levels of intensity are coded in the range $[0,1]$. We denote the $3 \mathrm{D}$ OCT data by $I(X, Y, Z), X \in[1,496], Y \in[1,384], Z \in[1,120]$, and the en-face image extracted at the abscissa $X$ from $I$ by $I^{(X)}$. We also denote the gray level of the voxel $P(X, Y, Z)$ indifferently by $I(X, Y, Z), I^{(X)}(Y, Z)$ or $I(P)$.

\subsection{Features in en-face images ( $X$ planes)}

Pores appear as small dark spots in the X planes, more or less contrasted with respect to the surrounding tissues. They correspond to local minima of intensity in the $2 \mathrm{D}$ enface images and have an overall circular or ovoid shape. Morphological operations [7] are applied to enhance these features. We denote by $D_{r}$ a binary structuring element representing a disk of radius $r$, and by $\oplus, \ominus, \circ$ and $\bullet$ the dilation, erosion, opening and closing operations, respectively. We propose the following preprocessing algorithms to enhance the pore features:

- A bottom-hat filter with a disk of radius $r=3$ in order to enhance the dark spots larger than the structuring element. We denote the negative of this image by $I_{B H}$ :

$$
I_{B H}^{(X)}=1-\left(\left(I^{(X)} \bullet D_{3}\right)-I^{(X)}\right)
$$

- Alternate sequential filters, which are defined by a sequence of closings and openings with increasing size of the structuring element $D_{i}$ (up to $i=3$ ), including morphological reconstruction at each step $i$. With the initialization $I_{\text {fas }}^{(X)(0)}=I^{(X)}$,

$$
\begin{aligned}
& I_{\text {open }}=R_{I_{\text {fas }}^{(X)(i-1)}}^{D}\left(I_{\text {fas }}^{(X)(i-1)} \circ D_{i}\right) \\
& I_{\text {fas }}^{(X)(i)}=R_{I_{\text {open }}}^{E}\left(I_{\text {open }} \bullet D_{i}\right)
\end{aligned}
$$

where $R_{M}^{D}(I)$ and $R_{M}^{E}(I)$ are respectively the reconstruction by dilation and by erosion of the image $I$ in the mask $M$. This allows denoising of the image while retaining the main structures, larger than the maximal size $(i=3)$ of the structuring elements. The subtraction of the input image reveals the main pores:

$$
I_{\text {FAS }}^{(X)}=1-\min \left(\max \left(I_{\text {fas }}^{(X)(3)}-I^{(X)}, 0\right), 1\right)
$$


Figure 2 shows the preprocessed en-face images. The pores appear as dark spots, of higher contrast than in the original image. It is worth noting that 3D morphological operations, experimentally tested, did not provide the best results in our final segmentations.
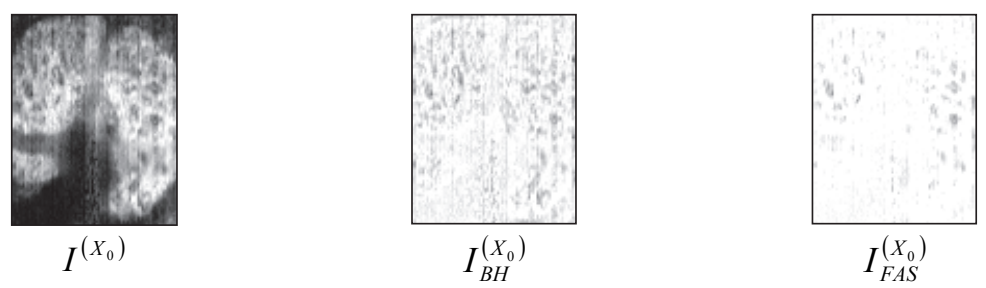

Fig. 2. Image preprocessing enhancing structural features of the pores.

\subsection{Features in the transverse images ( $\mathrm{Y}$ and $\mathrm{Z}$ planes)}

Axon paths present properties of regularity. First, the intensity of a path must keep almost the same gray level from one en-face image of abscissa $X$ to the next one, at $X+1$. Moreover, axon paths appear as vertical curves that are quite regular. It means that the $Y$ and $Z$ coordinates of a pore cannot vary a great deal from one $X$ plane to the next one. Axons also never remain in the same $X$ plane, they are globally oriented downwards. So we can design tracking algorithms that predict the position of a pore in the $X+1$ plane from its coordinates in the previous planes, $X, X-1, \ldots$ We propose two ways to do this. First, we designed a first-order recursive filter that provides an estimation of the pore coordinates in the $X+1$ plane from its coordinates in the previous $X$ planes. Second, we propose a parametric active contour model that enables us to find the local evolution of any axon path around a given position $(X, Y, Z)$. The snake is initialized by a small vertical segment centered on $(X, Y, Z)$. The minimization of an energy functional deforms the snake towards the local minima of intensity in the neighboring en-face images and provides a good estimation of the local shape of the path.

\section{$4 \quad$ Methods}

We present here the main methods that are involved in our lamina cribrosa segmentation algorithm, which will then be detailed in Section 5 .

\subsection{Extraction of the pores in a reference en-face image}

Let us denote by $X_{0}$ the abscissa of the en-face image where axon paths are initialized. This plane is currently manually selected (Section 5). We first extract the local minima of intensity in $I^{\left(X_{0}\right)}$, denoted by $M_{l}^{\left(X_{0}\right)}, l \in\left[1, L^{\left(X_{0}\right)}\right]$. For that, we calculate the cost function (4) at all points $M_{l}^{\left(X_{0}\right)}$ and sort them by increasing cost. 


$$
E\left(X_{0}, l\right)=\left(1-\alpha_{I P}\right) I_{F A S}^{\left(X_{0}\right)}\left(M_{l}^{\left(X_{0}\right)}\right)+\alpha_{I P} I_{B H}^{\left(X_{0}\right)}\left(M_{l}^{\left(X_{0}\right)}\right)
$$

We retain the first $N_{0}=25$ points that are distant by more than 5 pixels. The latter condition ensures that only one local minimum is selected per pore. Figure 3 illustrates this process: the main pores are actually retained as axon path initialization.
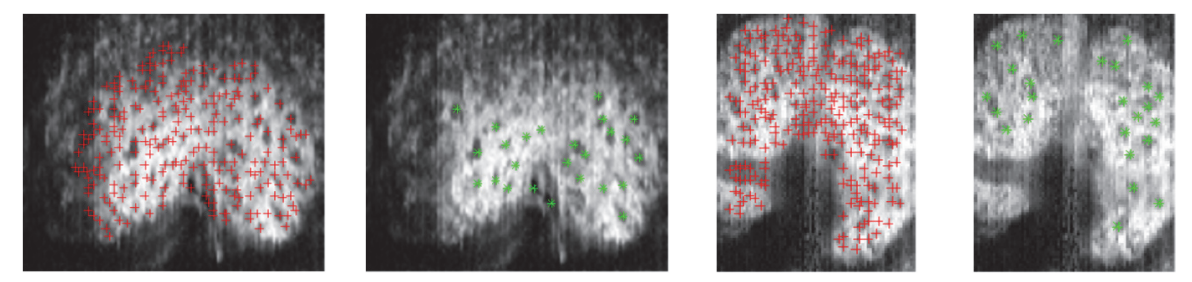

Fig. 3. Initialization of the pores in the reference $X$ plane for two different data sets. In red the local minima, in green the local minima selected as pores.

\subsection{Prediction model}

Let us consider one axon path $A_{n}, n \in\left[1, N_{0}\right]$, defined from the abscissa $X_{0}$ to the current position $X_{c}>X_{0}$ by a series of points $P_{n}^{(X)}\left(X, Y_{n}^{(X)}, Z_{n}^{(X)}\right)$, with $X \in\left\{X_{0}, X_{0}+\right.$ $\left.1, \ldots, X_{c}\right\}$. A first-order recursive filter is used to predict the next position $\hat{P}_{n}^{\left(X_{c}+1\right)}\left(X_{c}+1, \hat{Y}_{n}^{\left(X_{c}+1\right)}, \hat{Z}_{n}^{\left(X_{c}+1\right)}\right)$ of $A_{n}$ in the plane $X_{c}+1$ from the previous points:

$$
\left\{\begin{array}{l}
\hat{V}_{Y_{n}}^{\left(X_{c}\right)}=(1-\varphi)\left(Y_{n}^{\left(X_{c}\right)}-Y_{n}^{\left(X_{c}-1\right)}\right)+\varphi \hat{V}_{Y_{n}}^{\left(X_{c}-1\right)} \\
\hat{Y}_{n}^{\left(X_{c}+1\right)}=Y_{n}^{\left(X_{c}\right)}+\hat{V}_{Y_{n}}^{\left(X_{c}\right)}
\end{array}\right.
$$

where $\hat{Y}_{n}^{(X)}$ denotes the predicted $Y$ coordinate of the axon $A_{n}$ in the plane $X$. The initial speed $\widehat{V}_{Y_{n}}^{\left(X_{0}\right)}$ is set to 0 . This same model applies to the $Z$ coordinate. Thus we express regularity properties of axon paths: the current displacement in one direction, $\mathrm{Y}$ or $\mathrm{Z}$, is estimated from the previous displacements, which are continuously integrated in the filter. The higher the value of $\varphi$, the greater the number of points involved and the stronger the smoothing.

\subsection{Parametric snake model}

We propose a parametric snake model derived from the classic snake of Kass [8]. It consists of a parametric curve $V$, whose evolution is driven by the minimization of an energy function $E(V)(6)$ which includes terms controlling the smoothness of the curve (internal energy) and terms related to image data (image energy). Here we want a vertical segment to move towards the axon paths characterized by low intensity.

$$
E(V)=\int_{X_{1}}^{X_{2}} \alpha\left|V^{\prime}(s)\right|^{2}+\beta\left|V^{\prime \prime}(s)\right|^{2}+I(V(s)) d s
$$


The first two terms of (6) impose the curve $V$ to be smooth, while the third term attracts the curve towards the low intensities. The parameters $\alpha, \beta$ weight the relative importance of all terms. In our application, the parameter $s$ takes the discrete values $i=X_{1}, X_{1}+1, \ldots, X_{2}$, so that each point $i$ of the discretized curve $V$ moves horizontally in the en-face plane of abscissa $i$. The consistency and the smoothness of the curve are ensured by the internal energy term. The higher $\alpha$ and $\beta$ the straighter the curve $V$. The minima of $E$ are found by solving the following Euler-Lagrange equations:

$$
\left\{\begin{array}{l}
-\alpha Y^{\prime \prime}(s)+\beta Y^{\prime \prime \prime \prime}(s)+\frac{\partial I^{(s)}(Y(s), Z(s))}{\partial Y}=0 \\
-\alpha Z^{\prime \prime}(s)+\beta Z^{\prime \prime \prime \prime}(s)+\frac{\partial I^{(s)}(Y(s), Z(s))}{\partial Z}=0
\end{array}\right.
$$

where $Y(s)$ and $Z(s)$ are the coordinates of the curve $V(s)$ in the plane $s$. To solve equations (7), we classically introduce the time variable $t$ and discretize the equations as indicated previously, with one sample in each en-face image of abscissa $X \in\left\{X_{1}, \ldots, X_{2}\right\}$. This model is involved in the second tracking algorithm (Section 4.5) and in the final regularization of the axon pathways (Section 5).

\subsection{Tracking: method 1}

Let us denote by $M_{l}^{(X)}, l \in\left[1, L^{(X)}\right]$, the $L^{(X)}$ local minima of intensity in the en-face image of abscissa $X$. We assume that we have defined $N_{0}$ pores in the plane of abscissa $X_{0} \in\left[X_{\text {Start }}, X_{\text {Stop }}\right]$, according to the method presented in section 4.1. Our goal is now to track the pores from each of these $N_{0}$ positions, for the increasing $X$ coordinates and then for the decreasing $X$ coordinates, in order to make up $N_{0}$ axon paths, vertically defined in the range $\left[X_{\text {Start }}, X_{\text {Stop }}\right]$. Let us consider one axon path $A_{n}$, $n \in\left[1, N_{0}\right]$, defined from the abscissa $X_{0}$ to the current position $X_{c}>X_{0}$ by a series of points $P_{n}^{(X)}\left(X, Y_{n}^{(X)}, Z_{n}^{(X)}\right), X \in\left\{X_{0}, X_{0}+1, \ldots, X_{c}\right\}$. We want now to define the next position $P_{n}^{\left(X_{C}+1\right)}$ at $\mathrm{X}_{\mathrm{c}}+1$. Our first approach consists in the selection of $P_{n}^{\left(X_{c}+1\right)}$ among the candidates $M_{l}^{\left(X_{C}+1\right)}, l \in\left[1, L^{\left(X_{c}+1\right)}\right]$ (Fig. 4) via the minimization of a cost function, evaluating the structural features listed in Section 3. This cost function is at first made of two terms:

- A term which expresses the radiometric properties of the pores, which are supposed to be dark spots and present some continuity in the gray levels:

$$
E_{R}(n, l)=\alpha_{D} I_{F A S}^{\left(X_{c}+1\right)}\left(M_{l}^{\left(X_{c}+1\right)}\right)+\alpha_{S}\left|I_{B H}^{\left(X_{c}+1\right)}\left(M_{l}^{\left(X_{c}+1\right)}\right)-I_{B H}^{\left(X_{c}\right)}\left(P_{n}^{\left(X_{c}\right)}\right)\right|
$$

- A geometric term that expresses the smoothness of the axon paths, by calculating the Euclidian distance $d$ between the predicted position (5) and a candidate:

$$
E_{P}(n, l)=\alpha_{P} d\left(\hat{P}_{n}^{\left(X_{c}+1\right)}, M_{l}^{\left(X_{c}+1\right)}\right)
$$


Then, the local minimum $M_{l_{o p t}}^{\left(X_{c}+1\right)}$ is retained as next position $P_{n}^{\left(X_{c}+1\right)}$ for the axon path $A_{n}$ through the minimization of the global cost function:

$$
l_{\text {opt }}=\arg \min _{l}\left\{E_{R}(n, l)+E_{P}(n, l)\right\}
$$

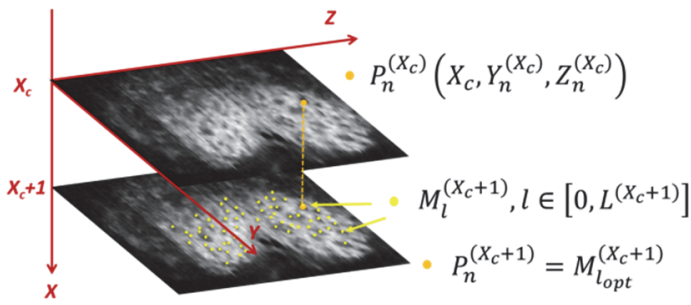

Fig. 4. Tracking of the pores from one plane to the next one.

In order to improve the robustness of this tracking algorithm, we add a preprocessing that consist of an evaluation of the spatial consistency of all local minima $M_{l}^{(X)}$. We apply the tracking algorithm (10) from every local minimum $M_{l}^{(X)}$ of every plane $X$ to create short paths centered on $M_{l}^{(X)}$ and of length $2 D X+1$ (from $X$ to $X$ - $D X$ and from $X$ to $X+D X$, with $D X=3)$. Then we store in an accumulator $C(X, Y, Z)$ the number of times each local minimum is passed through, the maximum being $2 D X+1$ and the minimum being 1 . We then normalize $C$. Thus, a $C(X, Y, Z)$ value near 1 indicates that the local minimum of coordinates $(X, Y, Z)$ is likely to be a way point of an axon, while a value near 0 indicates that this local minimum is likely to be noise. We integrate this metric in the cost function of the tracking method. So, instead of applying (10), we minimize the new cost function (12), integrating a spatial consistency term (11):

$$
\begin{gathered}
E_{C}(n, l)=\alpha_{C} C\left(M_{l}^{\left(X_{c}+1\right)}\right) \\
l_{\text {opt }}=\arg \min _{l}\left\{E_{R}(n, l)+E_{P}(n, l)+E_{C}(n, l)\right\}
\end{gathered}
$$

\subsection{Tracking method 2}

This second approach is based on the snake model presented in Section 4.3. Starting again from a reference plane $X_{0}$ where $N_{0}$ pores have been segmented, we initialize at each pore position $P_{n}^{(X)}\left(X, Y_{n}^{(X)}, Z_{n}^{(X)}\right)$ a small vertical segment centered on this position $([X \pm d X])$ and apply the snake algorithm. Each small snake deforms itself during a small number of iterations to reach the smallest intensities. Then we select the local minimum that is the nearest to the snake position in the adjacent plane, at $X+1$ or $X-1$. We iterate to get the complete axon paths, starting from the reference plane $X_{0}$, for the increasing and decreasing $X$ coordinates. This approach is less local than the previous one and so we can expect more robust and accurate results. 


\section{$5 \quad$ Axon path segmentation}

We can now describe the whole algorithm (Fig. 5) for the axon pathway segmentation from 3D SD-OCT data. The region of interest is manually defined by the user, who vertically bounds the ROI by selecting $X_{\text {Start }}$ and $X_{\text {Stop }}$, the abscissas inside which the axon pathways are generally visible enough to be processed. The user also defines the reference plane $X_{0}$, corresponding to the highest contrast en-face image, where the pores are the most obvious. The user delineates a mask in this reference plane where the pores can be searched for at any $X$ abscissa. The next steps are fully automatic.

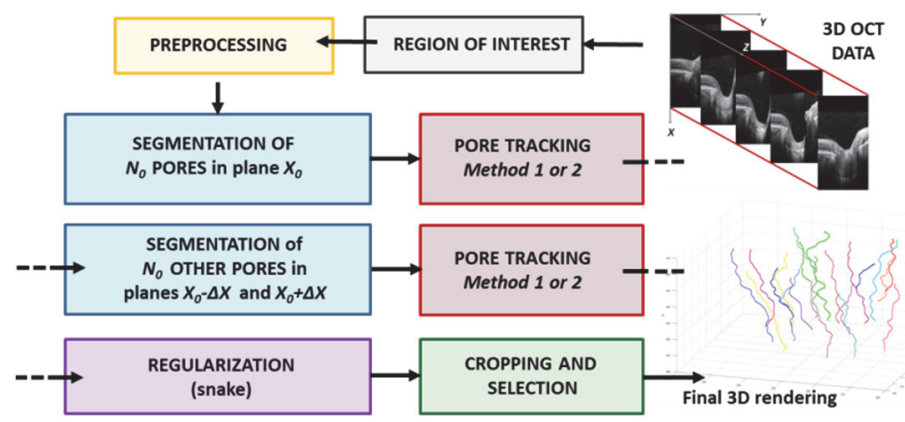

Fig. 5. Functional diagram of the segmentation method

The OCT data is then preprocessed (Section 3.1) and $N_{0}=25$ pores are extracted in the reference plane $X_{0}$ according to the method described in Section 4.1. The axon paths are segmented based on the tracking algorithm presented either in Section 4.4 or in Section 4.5, along the decreasing abscissas up to $X_{\text {Start }}$ and along the increasing abscissas up to $X_{\text {Stop. }}$. Then, $N_{0}=25$ other pores are segmented in the planes $X_{0}-\Delta X$ and $X_{0}+$ $\Delta X(\Delta X=10)$, according to the minimization of the cost function (4) over both planes simultaneously, under the constraint that the new pores must be distinct from the ones already segmented. The tracking procedure is again applied from these new pores. Finally, we get 50 axon paths, which are refined and regularized with the snake algorithm (Section 4.3). It is worth noting that the paths can cross each other or merge, which is anatomically possible, according to current thinking.

The parameters of the algorithm were optimized with respect to manual segmentations performed by an engineer specialized in image processing, on a learning database of 7 3D SD-OCT images. We obtained this set of parameters:

- Pore segmentation (4): $\alpha_{I P}=0.05$

- Prediction model (5): $\varphi=0.95$

- Tracking algorithm $1(8)(9)(11): \alpha_{D}=12 ; \alpha_{S}=1 ; \alpha_{P}=1 ; \alpha_{C}=4$;

- Snake model (7): $\alpha=0.05, \beta=0$ :

- Tracking algorithm, method 2: $d X=3$;

Figures 6, 7 and 8 show examples of segmentations. For the transverse planes, the gray levels have been calculated by interpolation to take into account the path trajectory in 
the orthogonal direction (note that the background appearance is not exactly the same in Fig.7(a) versus (b) and (c) versus (d) for that reason).
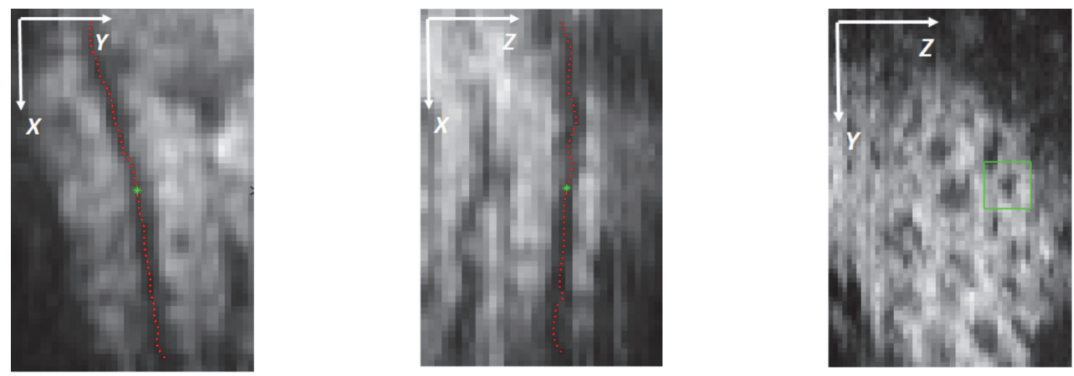

Fig. 6. Example of automatic segmentation of an axon path in a high contrast case.

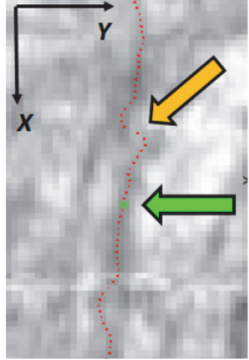

(a)

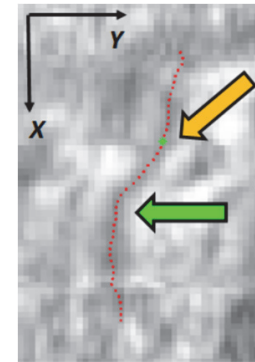

(b)

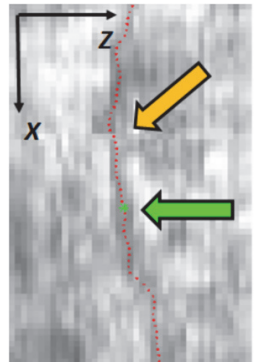

(c)

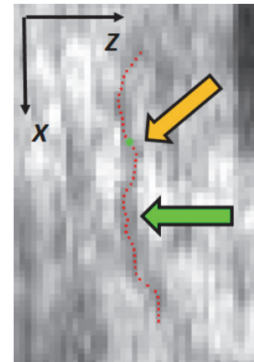

(d)

Fig. 7. Example of axon segmentation. (a)(c) Automatic segmentation provided by our method; (b)(d) manual segmentation provided by a medical expert. There is a good match at the green arrows, and a divergence at the orange arrows.

In Figure 6, the image is high contrast and the path is determined without ambiguity. The case in Figure 7 is much more difficult and there are places where it is difficult to know where the path is headed. In this example, the algorithm diverges compared to the manual segmentation at the orange arrow $(X \sim 383)$. For a greater abscissa $(X \sim 393)$, the path is higher contrast and there is a good match between the automatic and manual segmentations. Note that the transverse images in Figures 7(a) and (b), and in Figures 7(c) and(d), are different as these images are reconstructed at each X coordinate given the path position in the direction orthogonal to the considered plane. An overall impression of continuity of the pixel intensities in the transverse reconstructed images is generally associated with a non-ambiguous segmentation of the axon pathway. Figure 8 illustrates the same example, by showing the positions of the pore with respect to the decreasing $\mathrm{X}$ coordinate, for the manual and automatic segmentations. The position found by the automatic algorithm slightly shifts around $X=383$, where two different pores touch each other, and finally follows the wrong path. This example illustrates the difficulty of the segmentation task, due to the image noise, the poor contrast and the low resolution given the pore size. 


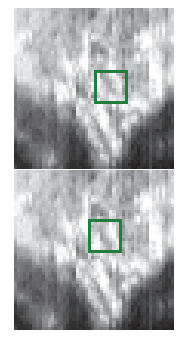

$X=385$

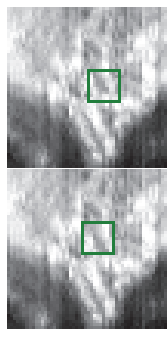

$X=384$

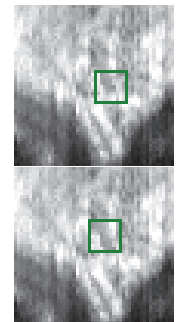

$X=383$

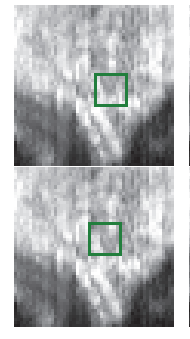

$X=382$

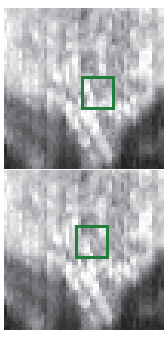

$X=381$

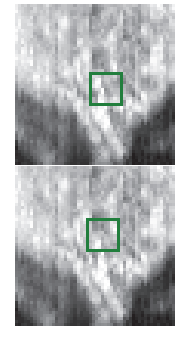

$X=380$

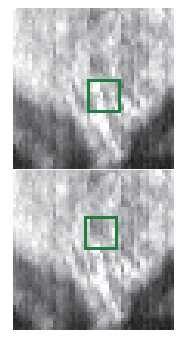

$X=379$

Fig. 8. An example of failure in the pore tracking with respect to the decreasing $X$ coordinate. The first row shows the manual segmentation and the second the automatic segmentation.

The last two steps concern the cropping of axonal paths, which are often not visible from end to end, and the selection of those paths which are most relevant. These steps are performed by thresholding a cost function similar to the function involved in pore tracking (Section 4.4). The thresholds are set dynamically in each image.

\section{Experiments and quantitative evaluation}

Experiments were conducted on a database of 21 3D SD-OCT images coming from 15 patients. This database was fully segmented by an engineer (ENG) and 7 segmented images were involved in the parameter learning. The other 14 images were also segmented by an ophthalmologist (EXP), who is an expert in glaucoma diseases, and this subset constitutes the test set. The aim of the ophthalmologist was not to extract exhaustively all the axon paths, but rather to segment the most relevant ones with regards to clinical studies. The quantitative evaluation is done on the test set.

We denote a reference segmentation taken as ground truth by $G T$, and a segmentation to be evaluated with respect to GT by TEST. The first part of the quantitative evaluation concerns the detection of the axon paths. We simply calculate the number of true positives (TP, number of paths present in GT and also detected in TEST), the number of false positives (FP, extra paths of TEST with no equivalent in $G T$ ) and the number of false negative (FN, paths of $G T$ with no equivalent in TEST). We consider that two paths provided by two different segmentations (GT and TEST) match if the distance between both is less than 2 pixels on at least $20 \%$ of the path of $G T$.

The second part of the evaluation concerns the accuracy of the TP paths. Let us consider two matching paths, $A_{n}^{(G T)}$ and $A_{n}^{(T E S T)}$, and let us denote by $I_{X, n}$ the X interval of overlapping. All points of $A_{n}^{(T E S T)}$ inside $I_{X, n}$ are counted as true positive points (tp) whatever their distance with their equivalent (same abscissa) in $A_{n}^{(G T)}$. The points of path $A_{n}^{(G T)}$ outside $I_{X, n}$ are false negative points (fn) and the points of $A_{n}^{(T E S T)}$ outside $I_{X, n}$ are false positive points (fp). Finally, we calculate the root mean squared error (RMSE) between $A_{n}^{(G T)}$ and $A_{n}^{(T E S T)}$ on $I_{X, n}$ to evaluate the accuracy of the tested segmentation with respect to the ground truth. All these measurements are averaged over 
images and paths to provide a statistical evaluation on the test set. We also indicate the mean path depth (i.e. mean number of points in a path) to help interpretation.

Table 1. Comparison between the manual segmentations performed by the medical expert (EXP), taken as ground truth, and the manual segmentations performed by the engineer $(E N G)$.

\begin{tabular}{|l|l|l|}
\hline Mean path depth (EXP): & \multicolumn{2}{l|}{$26.12+/-11.12$ points } \\
Mean path depth (ENG): & $37.11+/-14.06$ points & \\
\hline TP (paths) & FN (paths) & FP (paths) \\
$62(56.36 \%)$ & $48(43.64 \%)$ & $184(167.27 \%)$ \\
\hline tp (points) & fn (points) & fp (points) \\
$22.16+/-8.74$ & $4.63+/-6.67$ & $15.85+/-12.67$ \\
RMSE $=\mathbf{1 . 7 8}+/-\mathbf{1 . 4 1}$ pixels & \\
\hline
\end{tabular}

Table 2. Evaluation of the automatic method (AUTO) with respect to the medical expert's segmentations (EXP), for the two tracking algorithms (1 in black, 2 in blue).

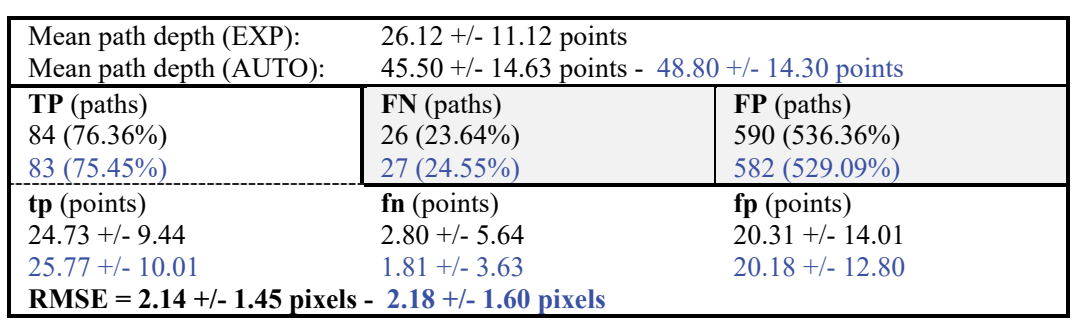

We first compare the manual segmentations provided by the engineer $(E N G)$ and the ones performed by the medical expert $(E X P)$, taken as ground truth (Table 1). The engineer segmented many more axonal pathways than the expert (246 against 110), but only half of the expert's pathways are also processed by the engineer. This is due to the difficulty of exploring the OCT volume and interpreting it. The engineer segmented also the axonal pathways on larger depths (37 points against 26 in average); the overlap is very good for the TP paths, $85 \%$ of the reference path in average, with a RMSE less than 2 pixels. To summarize, we observe a very good match between both manual segmentations on their common part, but a significant difference in the detection and selection of the axonal pathways to be segmented, probably due to differences in the interpretation criteria.

Table 2 shows the quantitative evaluation of the automatic algorithm (AUTO) with respect to the expert's ground truth, for both variants (tracking method 1 or 2). We get very similar results for both tracking approaches. The automatic algorithm segments many more axon pathways than the expert. We also observe an overall oversegmentation on the TP paths with an average of $\sim 20$ extra points. The number of false negatives is low, so the overlap is good ( $95 \%$ of the reference path in average). The RMSE values are very good, close to the EXP/ENG difference. The comparison of the automatic algorithm with respect to the engineer's segmentations leads to the following results for the first variant of the algorithm: $89 \%$ of TP paths, with $181 \%$ of FP; on the TP paths, an overlap of $91 \%$ with a RMSE $=1.23+/-1.16$ pixels; $12.30+/-12.15$ of extra (fp) points, in average. The results for the second variant are similar. 


\section{$7 \quad$ Conclusion and perspectives}

We have proposed a segmentation method of the lamina cribrosa acquired in 3D SDOCT. To the best of our knowledge, it is the first time such segmentation has been attempted. Our method is based on the modeling of radiometric and structural features of the pores, embedded in detection and tracking algorithms. Our first experimental evaluation shows very interesting results, as the true positive pathways $(76 \%$ of the reference paths) are accurately segmented with an RMSE in the range of the inter-expert segmentation difference. Both tracking algorithms led to very similar results; so the first one will probably be selected as it is faster and easier to integrate in user-guided procedures, which can be interesting in complement to the fully automatic algorithm. However we face difficulties in interpreting the 3D OCT data even manually. To overcome this, we need to involve several medical experts, willing to perform manual segmentation as exhaustively as possible, so as to accurately learn axon path features and establish a ground truth.

This tridimensional analysis is both innovative and promising, allowing for the first time the observation of the possible causes of neuronal loss during glaucoma. Longitudinal follow-up will be a key element in determining disease progression. A comparison with reference examinations (visual field) will be necessary to determine to what extent this approach is interesting in the management of patients.

\section{References}

1. S. Zwillinger, M. Paques, B. Safran, C. Baudouin, In vivo characterization of lamina cribrosa pore morphology in primary open-angle glaucoma, Journal Français d'Ophtalmologie, Vol. 39, Issue 3, pp 265-271, 2016.

2. C. Burgoyne, The morphological difference between glaucoma and other optic neuropathies. Journal of Neuro-ophthalmology, 2015,35(0 1):S8-S21.

3. Mei Hui Tan, S. H. Ong, S. G. Thakku, et al., Automatic Feature Extraction of Optical Coherence Tomography for Lamina Cribrosa Detection. Journal of Image and Graphics, 2015, vol. 3, no 2 .

4. K. Omodaka, T. Horii, S. Takahashi, T. Kikawa, et al., 3D Evaluation of the Lamina Cribrosa with Swept-Source Optical Coherence Tomography in Normal Tension Glaucoma. PLoS ONE 10(4): e0122347, 2015.

5. A. Belghith, C. Bowd, F. A. Medeiros, R. N. Weinreb and L. M. Zangwill, Automated segmentation of anterior lamina cribrosa surface: How the lamina cribrosa responds to intraocular pressure change in glaucoma eyes?, IEEE ISBI conference, New York, NY, pp. 222225,2015

6. Z. Nadler, B. Wang, G. Wollstein, et al. Automated lamina cribrosa microstructural segmentation in optical coherence tomography scans of healthy and glaucomatous eyes. Biomedical Optics Express. 2013;4(11):2596-2608, 2013.

7. R. C. Gonzalez and R. E. Woods. Digital Image Processing (3rd Edition), Chapt. 9. Ed. Prentice-Hall, 2006.

8. M. Kass, A. Witkin, and D. Terzopoulos, Snakes: Active contour models, International Journal of Computer Vision, vol. 1, no. 4, pp. 321-331, 1988. 